\section{Propiedades psicométricas del inventario de depresión de Beck IA para la población chilena}

\author{
CAMILA VALDÉS ${ }^{1, a}$, IRMA MORALES-REYES ${ }^{1, b}$, \\ J. CAROLA PÉREZ ${ }^{2, c}$, ADRIANA MEDELLÍN ${ }^{3, d}$, \\ GRACIELA ROJAS ${ }^{4}$, MARIANE KRAUSE ${ }^{1, \mathrm{e}}$
}

\section{Psychometric properties of a spanish version of the Beck depression inventory IA}

Background: According to the Chilean National Health Survey (20092010), 17\% of people aged 15 years or more have depressive symptoms. Thus, freely-available, easily-administered, and highly sensitive screening tests for depression are needed in clinical and research settings. Aim: To evaluate the psychometric properties of a Spanish version of the Beck Depression Inventory (BDI-IA) in adult Chilean population. Material and Methods: The inventory was applied to a sample of 1.105 adults aged between 18 to 73 years ( $94 \%$ women). Ninety nine participants were outpatients receiving treatment for affective disorders, 932 were parents and/or guardians of students enrolled in schools and 73 were university students (sample with no known depressive disorder). To perform data analysis, two groups from the random combination of both samples were generated. Results: The inventory showed an appropriate degree of internal consistency (Cronbach alpha =.92). An exploratory factor analysis suggested a one-factor solution. This solution was reinforced with a confirmatory factor analysis, which displayed an adequate goodness offit. The cutoff score, based on the Youden Index, was 13/14 points. It was able to discriminate between depressed and non-depressed participants. Conclusions: These results indicate that the BDI-IA is an appropriate instrument to assess depressive symptoms in Chilean adults.

(Rev Med Chile 2017; 145: 1005-1012)

Key words: Depression; Psychometrics; Surveys and Questionnaires.
'Escuela de Psicología, Pontificia Universidad Católica de Chile. Santiago, Chile.

${ }^{2}$ Facultad de Psicología, Universidad del Desarrollo. Santiago, Chile.

${ }^{3}$ Departamento de Salud Mental, Hospital de Curacaví. Curacaví, Chile. ${ }^{4}$ Hospital Clínico, Facultad de Medicina, Universidad de Chile. Santiago, Chile. aLicenciada en Ciencias Biológicas, estudiante Programa de Doctorado en Psicología, Pontificia Universidad Católica de Chile. Santiago, Chile. bPsicóloga, estudiante Programa de Doctorado en Psicoterapia, Pontifica Universidad Católica de Chile. Santiago, Chile.

'Psicóloga, Magíster en Psicología Clínica, Doctor en Psicología.

'Psicóloga, estudiante Programa de Magister en Psicología de la Salud,

Pontificia Universidad Católica de Chile. Santiago, Chile.

ePsicóloga, Doctor en Psicología.

Financiamiento: Este estudio recibió apoyo del Fondo de Innovación para la Competitividad (FIC) del Ministerio de Economía, Fomento y Turismo, a través de la Iniciativa Científica Milenio, Proyecto IS130005 y Proyecto NS100018. Asimismo, conto con financiamiento del proyecto FONDECYT 11130041.

Recibido el 27 de enero de 2017 , aceptado el 29 de agosto de 2017

Correspondencia a:

J. Carola Pérez

Instituto Milenio para la Investigación en Depresión y Personalidad.

Oficina 202, Segundo piso MIDE-UC, Av. Vicuña Mackenna 4860, Macul,

Santiago, Chile.

janetperez@udd.cl

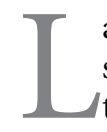

a depresión es una alteración del ánimo que se expresa a través de una serie de manifestaciones sintomáticas que incluyen tristeza patológica, anhedonia, irritabilidad, sentimientos de culpa, pesimismo y desesperanza ${ }^{1}$. Deteriora la calidad de vida de las personas que la padecen, así como la de su entorno familiar y social ${ }^{2}$. Si bien existe consenso respecto a lo anterior, no hay una conceptualización unívoca de la etiología y tipología de la depresión.

De acuerdo a la Encuesta Nacional de Salud ${ }^{3}$, realizada entre los años 2009 y 2010, la prevalencia de síntomas depresivos en la personas mayores de 15 años es de $17,2 \%$, valor que aumenta a $25,7 \%$ si se considera sólo a las mujeres. Según los datos epidemiológicos arrojados por el estudio de Vicente y cols. en el año 2002, la prevalencia de vida de depresión alcanza a $9 \%$ en las mujeres y $6,4 \%$ en los hombres ${ }^{4}$. De acuerdo al estudio de carga de enfermedad y carga atribuible, la depresión unipolar es la segunda causa de años de vida perdidos ajustados por discapacidad en población general en Chile y la primera en mujeres entre 20 y 44 años $^{5}$. Entre los años 2008 y 2009, los tras- 
tornos mentales y del comportamiento generaron el mayor gasto por concepto de licencias médicas en el país $(20,7 \%)$, y dentro de estos trastornos, la depresión corresponde a 59,3\% ${ }^{6}$.

La detección confiable de la depresión es necesaria para su abordaje oportuno y tratamiento apropiado, acciones esenciales para disminuir la carga de enfermedad que este trastorno representa. Es por esto que el uso de instrumentos que evalúen la sintomatología depresiva de forma dimensional es de vital importancia para las tareas de cribado y diagnóstico, tanto en muestras clínicas como en la población general ${ }^{7}$.

El inventario de depresión de Beck I (internacionalmente conocido como BDI-I) es una de las escalas más utilizadas en el mundo para medir la severidad de los síntomas de depresión en muestras clínicas y no clínicas ${ }^{8}$. Las propiedades psicométricas del instrumento han sido reportadas en un artículo que integra los resultados de estudios realizados con este inventario durante 25 años, mostrando que posee características psicométricas adecuadas $^{8}$.

El presente estudio tuvo como objetivo evaluar las características psicométricas del BDI-IA en la población chilena, en términos de su consistencia interna, su estructura factorial, y para establecer el puntaje de corte que permita distinguir entre una muestra clínica y una muestra no clínica respecto a la patología depresiva.

\section{Material y Métodos}

\section{Muestra}

La muestra total estuvo compuesta por 1.105 adultos (18-73 años, 94\% mujeres) provenientes de tres estudios independientes, desarrollados en el marco del Instituto Milenio para la Investigación en Depresión y Personalidad (MIDAP).

La muestra estuvo conformada por un grupo de 99 consultantes (18-68 años, 78\% mujeres) de un servicio de salud ambulatorio, quienes presentaban un diagnóstico clínico de depresión, un grupo de 73 estudiantes universitarios (18-24 años, $62 \%$ mujeres) y 932 apoderados de establecimientos educacionales (26-73 años, 97\% mujeres) sin diagnóstico conocido, y quienes reportaron no encontrarse en tratamiento psicológico o farmacológico por depresión u otro trastorno de salud mental.
Estas muestras fueron combinadas para conformar dos nuevos mixtos (que incluyeron personas con diagnóstico de depresión como aquellos sin patología diagnosticada). Esta decisión se tomó considerando que es una estrategia sugerida para realizar análisis factorial cuando las muestras son muy disímiles entre sí $i^{9}$ Los grupos (ver Tabla 1) pueden ser considerados homogéneos respecto a las variables género, edad y escolaridad ( $\left.\mathrm{p}_{\mathrm{s}}>0,05\right)$.

\section{Instrumentos}

El inventario de depresión de Beck I (BDI-IA) es un cuestionario de autorreporte desarrollado para medir la gravedad subjetiva de la sintomatología depresiva, durante la última semana ${ }^{10}$. Consta de 21 ítems, con cuatro opciones de respuesta ( 0 a 3 puntos), donde un mayor puntaje representa mayor sintomatología depresiva ${ }^{10}$. En este estudio se utilizó la versión adaptada por el equipo MIDAP, construida a partir de la versión española ${ }^{11}$, la cual fue modificada en función del lenguaje más comúnmente usado en Chile.

\section{Procedimientos}

Cada estudio fue autorizado por el Comité de Ética de la institución respectiva. El Comité de Psicomédica Research Group autorizó el estudio con el grupo de consultantes con diagnóstico de depresión, mientras que los comités de ética de las escuelas de Psicología, de la Pontificia Universidad Católica de Chile y de la Universidad del Desarrollo, autorizaron los estudios en los contextos universitario y escolar.

Los participantes aceptaron voluntariamente formar parte de los estudios y firmaron el consentimiento informado correspondiente a cada uno de ellos. El inventario BDI-IA se aplicó como uno de los cuestionarios de la batería específica de instrumentos de cada estudio. En el grupo con diagnóstico de depresión, la aplicación fue realizada antes del inicio del proceso psicoterapéutico en forma individual, completando el paciente el cuestionario en el contexto de las entrevistas iniciales. En los grupos sin diagnóstico conocido, la modalidad de aplicación fue individual (apoderados) o colectiva (estudiantes universitarios).

Como se mencionó anteriormente, los grupos se combinaron aleatoriamente para generar dos grupos mixtos. El primer grupo mixto se utilizó para establecer una solución factorial inicial (análisis factorial exploratorio $[\mathrm{AFE}] \mathrm{n}=536$ ) y el 
Inventario de depresión de Beck IA en población chilena - C. Valdés et al

Tabla 1. Descripción de las muestras

\begin{tabular}{|c|c|c|}
\hline & Muestra Mixta 1 & Muestra Mixta 2 \\
\hline Edad (años) & $42,17 \pm 9,48$ & $41,31 \pm 9,28$ \\
\hline Sexo femenino (\%) & $93,7 \%$ & $93,5 \%$ \\
\hline \multicolumn{3}{|l|}{ Escolaridad } \\
\hline Básica incompleta & $0,8 \%$ & $0,5 \%$ \\
\hline Básica completa & $2,2 \%$ & $2,5 \%$ \\
\hline Media incompleta & $5,8 \%$ & $6,5 \%$ \\
\hline Media completa & $31,0 \%$ & $26,6 \%$ \\
\hline Técnico incompleto & $8,1 \%$ & $5,7 \%$ \\
\hline Universitaria incompleta o técnica completa & $28,2 \%$ & $32,2 \%$ \\
\hline Universitaria completa o superior & $23,9 \%$ & $26,0 \%$ \\
\hline \multicolumn{3}{|l|}{ Conformación muestra } \\
\hline Casos con depresión ( $n, \%)$ & $49 \quad(9,2 \%)$ & $50 \quad(8,8 \%)$ \\
\hline Estudiantes (n, \%) & $36 \quad(6,7 \%)$ & $38 \quad(6,7 \%)$ \\
\hline Apoderados (n, \%) & $451(84,1 \%)$ & $481(84,5 \%)$ \\
\hline $\mathrm{n}$ & 536 & 569 \\
\hline
\end{tabular}

segundo para determinar el ajuste de la solución factorial obtenida anteriormente (análisis factorial confirmatorio $[\mathrm{AFC}] \mathrm{n}=569$ ).

El AFE se desarrolló utilizando como método de extracción de factores el análisis de ejes principales, considerando que es una de las estrategias más idóneas cuando no se cumple la normalidad multivariada $^{12}$. Para determinar el número de factores a extraer se utilizó como criterio el test MAP de Velicer ${ }^{13}$. Adicionalmente, se extrajo el gráfico de sedimentación.

En función de los resultados del AFE, el AFC testeó el modelo de un solo factor. Dado que los ítems del instrumento no cumplen con el supuesto de normalidad multivariada y son de naturaleza ordinal, se utilizó Weighted Least Squares (WLS) como método de estimación, según lo recomendado en la literatura ${ }^{14}$. Como indicadores de ajuste del modelo, se consideraron diferentes índices: a) contraste de razón de verosimilitudes o estadístico $\chi^{2}$, el cual indica un buen ajuste del modelo cuando no es significativo $(\mathrm{p} \geq 0,05)^{15}$; b) raíz media cuadrática del error de aproximación (Root Mean Square Error of Approximation; RMSEA), donde valores RMSEA $\leq 0,05$ indicarían un buen ajuste, mientras que valores que fluctúen entre 0,05 y 0,08 indicarían un ajuste aceptable ${ }^{16}$; y c) índice de ajuste comparativo (Comparative Fit Index, CFI), donde valores $\mathrm{CFI} \geq 0,95$ indican un buen ajuste del modelo ${ }^{17}$. Posteriormente, se evaluó la invarianza factorial de la solución unifactorial, para determinar si esta solución era estable al considerar la variable sexo.

Finalmente, se calculó la curva Receiver Operating Characteristic (ROC), con el fin de determinar de qué manera el BDI-IA permitiría distinguir entre las personas con diagnóstico clínico de depresión y aquellas sin presencia de patología ${ }^{18}$. La curva ROC fue calculada con la totalidad de la muestra, obteniendo el área bajo la curva, análisis que indica qué tan buen indicador es el BDI-IA para distinguir entre ambos grupos. Además, se calculó el puntaje de corte óptimo mediante dos métodos: el método Youden ${ }^{19}$ y el método de cercanía al vértice superior izquierdo ${ }^{20}$. También se calculó la sensibilidad y la especificidad del puntaje de corte original $(9 / 10)^{10}$.

El AFE y la curva ROC fueron realizados en el software estadístico R.v3.1.2 ${ }^{21}$. Para la curva ROC se utilizó la librería pROC del mismo software estadístico $^{22}$. El análisis factorial confirmatorio fue realizado en el software estadístico MPlus.v $7^{23}$. 


\section{Resultados}

\section{Confiabilidad}

Consistencia interna. La consistencia interna del instrumento, medida a través del coeficiente alfa de Cronbach, fue de 0,92 para la muestra total con datos completos ( $\mathrm{n}=1.007,21$ ítems); alcanzando valores de $\alpha=0,91$ en el grupo mixto usado para el AFE y de $\alpha=0,92$ en el grupo mixto usado en el AFC.

\section{Validez}

Análisis factorial exploratorio. Se realizó un AFE extrayendo un solo factor de acuerdo a lo arrojado por el test MAP de Velicer ${ }^{13}$. En la Figura 1 se presenta el gráfico de sedimentación que apoya la elección de la solución de un solo factor. La varianza explicada por el modelo de un factor fue de $37 \%$. La Tabla 2 presenta la matriz de cargas factoriales, donde se observa que prácticamente la totalidad de los ítems presentaron cargas factoriales superiores a 0,3 ; valor que de acuerdo a la literatura se considera suficiente para indicar la pertenencia significativa a dicho factor ${ }^{24}$. Sólo el ítem S ("Pérdida de peso") presentó valores por debajo de lo adecuado $(0,20)$, desviándose del patrón antes mencionado.

Análisis factorial confirmatorio. Se realizó un AFC con el fin de confirmar la estructura de un solo factor del instrumento. El modelo presentó índices de ajuste buenos, dando cuenta de la estructura unifactorial del instrumento $\left(\chi^{2}(189\right.$, $\mathrm{n}=569)=463,53, \mathrm{p}=0,00$, RMSEA $=0,051$, CFI $=0,979)$. Las cargas factoriales de cada ítem se presentan en la Tabla 2.

Adicionalmente, se calculó la invarianza del modelo al considerar separadamente ambos sexos. Se encontró una diferencia de $\chi^{2}$ no significativa $\left(\Delta \chi^{2}=25,37, \mathrm{gl}=20, \mathrm{p}=0,19\right)$, lo que indicaría que no se encuentran diferencias en la estructura factorial entre hombres y mujeres.

\section{Puntaje de corte}

Curva ROC. Se calculó la curva ROC para la totalidad de la muestra tomando el puntaje total del BDI-IA, calculado como la suma de los puntajes en cada uno de los ítems y la separación entre el grupo con diagnóstico clínico de depresión y el grupo sin diagnóstico conocido. El gráfico asociado a esta curva puede observarse en la Figura 2. El área bajo la curva (AUC) calculada fue de 0,954. El punto de corte de 10, que corresponde al propuesto en la creación del instrumento ${ }^{10}$, presentó una especificidad de $80 \%$ y una sensibilidad del $92,86 \%$. El método de Youden entregó un punto de corte óptimo igual a 13,8, presentando una especificidad de $85,72 \%$ y una sensibilidad de $90,81 \%$. El puntaje de corte óptimo entregado por el método de

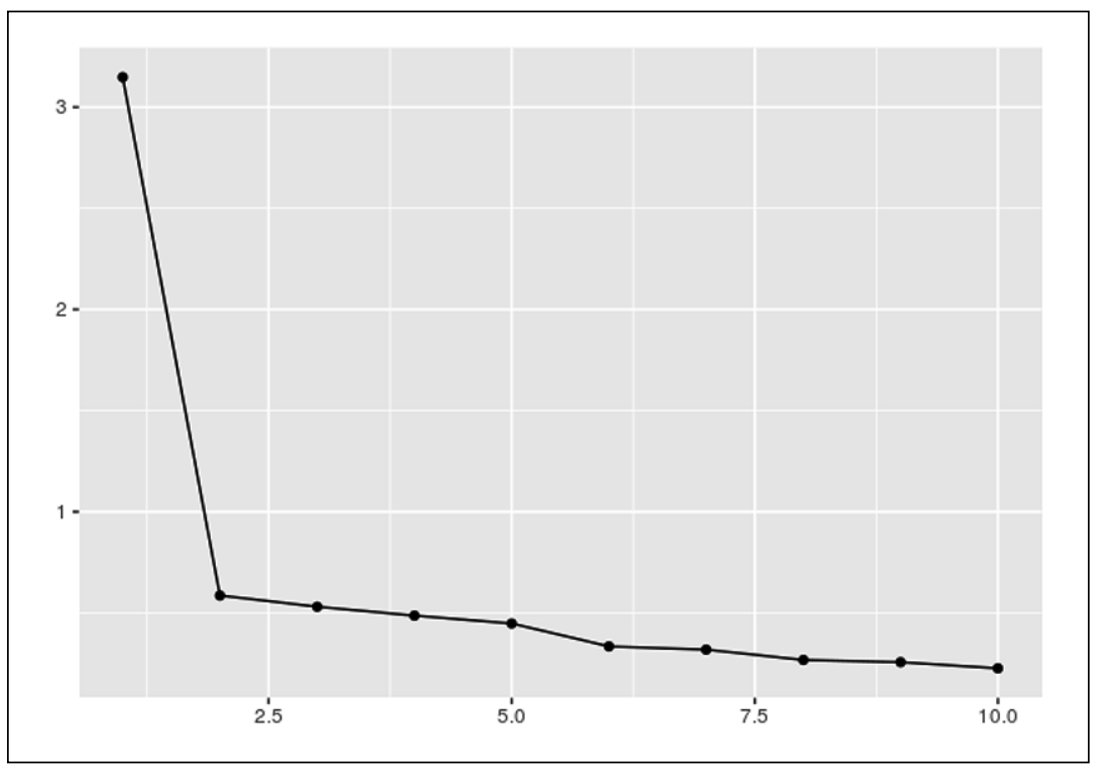

Figura 1. Gráfico de sedimentación del análisis factorial exploratorio. 
Inventario de depresión de Beck IA en población chilena - C. Valdés et al

Tabla 2. Cargas factoriales de la solución uni-factorial de los ítems del BDI-IA, según el análisis factorial exploratorio y análisis factorial confirmatorio

\begin{tabular}{|c|c|c|}
\hline ítem & Análisis factorial exploratorio & Análisis factorial confirmatorioa \\
\hline A: Tristeza & 0,76 & 0,72 \\
\hline B: Pesimismo & 0,72 & 0,77 \\
\hline C: Fracaso & 0,62 & 0,72 \\
\hline D: Satisfacción & 0,72 & 0,70 \\
\hline E: Culpa & 0,69 & 0,64 \\
\hline F: Castigo & 0,64 & 0,67 \\
\hline G: Decepción & 0,72 & 0,79 \\
\hline H: Autocrítica & 0,65 & 0,58 \\
\hline I: Ideación suicida & 0,62 & 0,77 \\
\hline J: Llanto & 0,64 & 0,72 \\
\hline K: Irritabilidad & 0,42 & 0,39 \\
\hline L: Interés & 0,57 & 0,56 \\
\hline M: Inseguridad & 0,66 & 0,66 \\
\hline N: Atractivo físico & 0,58 & 0,45 \\
\hline O: Iniciativa & 0,71 & 0,68 \\
\hline P: Sueño & 0,52 & 0,46 \\
\hline Q: Cansancio & 0,61 & 0,45 \\
\hline R: Apetito & 0,53 & 0,49 \\
\hline S: Pérdida de peso & 0,20 & 0,15 \\
\hline T: Preocupación por la salud & 0,47 & 0,32 \\
\hline U: Sexualidad & 0,53 & 0,35 \\
\hline
\end{tabular}

Nota: a Cargas Factorial Estandarizadas. Todos los ítems significativos $p<0,05$.

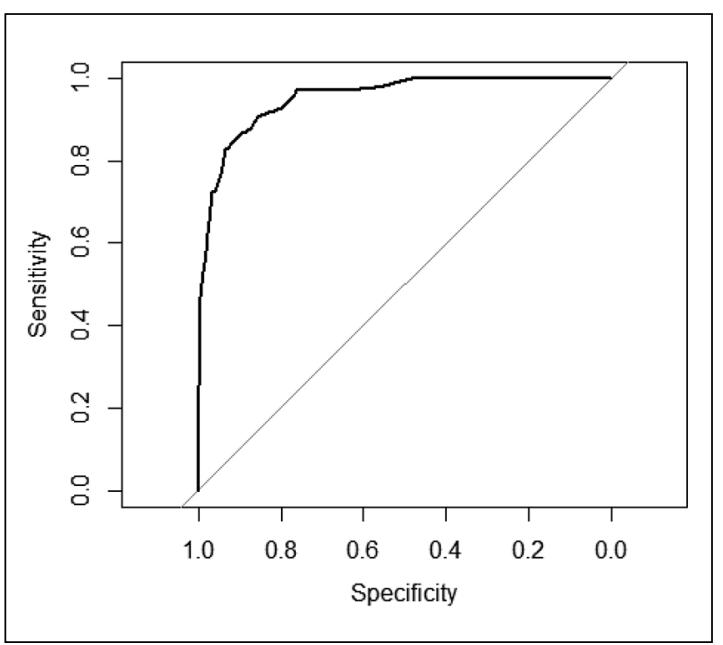

Figura 2. Curva ROC. cercanía al vértice superior izquierdo es de 11,5, presentando una especificidad de $85,73 \%$ y una sensibilidad de $86,73 \%$.

\section{Discusión}

Los resultados del presente estudio muestran que la adaptación chilena del BDI-IA posee una consistencia interna adecuada $(\alpha=0,92)$, muy similar a la reportada en la versión original $(\alpha=0,88)^{10}$ y a la revisión posterior del año 1979 $(\alpha=0,86)^{25}$. Del mismo modo, los resultados también son comparables con otras versiones del BDI-IA en español, como la versión mexicana $(\alpha=0,78)^{26}$; las versiones españolas reportadas por Ibáñez, Peñate, y González, $\alpha=73^{27}$, y Sanz 
y Vázquez, $\alpha=0,83^{11}$; la versión puertorriqueña, $\alpha=0,88^{28}$ y la versión argentina, $\alpha=0,80^{7}$. Estos valores indican que el BDI-IA es un instrumento confiable para detectar sintomatología depresiva, aportando evidencia que apoya su valor transcultural en tanto evalúa un síndrome de depresión general de síntomas interrelacionados, tal como lo definen Beck y cols. en su versión original del año $1961^{10}$, pudiendo ser usado en la población general.
Por otra parte, el instrumento mostró una estructural factorial que difiere de la solución propuesta la versión original ${ }^{10}$. En este estudio, la solución factorial más adecuada para el instrumento es la de un único factor de primer orden que engloba los 21 ítems que componen el inventario. Esta solución fue corroborada a través del AFC, cuyos resultados indican un buen ajuste del modelo. Otras versiones en español del instrumento (ver Tabla 3) presentaron soluciones diversas, como la

Tabla 3. Resumen de resultados de estudios con BDI-I en población hispanoparlante

\begin{tabular}{|c|c|c|c|c|c|}
\hline Estudio & $\begin{array}{l}\text { Características } \\
\text { de la muestra }\end{array}$ & $\begin{array}{l}\text { Versión } \\
\text { utilizada }\end{array}$ & $\begin{array}{l}\text { Análisis factorial } \\
\text { exploratorio }\end{array}$ & $\begin{array}{l}\text { Análisis factorial } \\
\text { confirmatorio }\end{array}$ & $\begin{array}{l}\text { Principales } \\
\text { resultados }\end{array}$ \\
\hline España ${ }^{27}$ & $\begin{array}{l}\mathrm{n}=393 \\
\text { Muestra no clínica } \\
61 \% \text { mujeres } \\
16-40 \text { años } \\
(\mathrm{M}=19,11 \\
\mathrm{DE}=2,79)\end{array}$ & $\begin{array}{l}\text { Versión española } \\
\text { de Conde et al. } \\
\text { (1996) } \\
19 \text { ítems }\end{array}$ & $\begin{array}{l}\text { n de factores: } \\
\text { Criterio Kaiser } \\
\text { (eigenvalue } \geq 1 \text { ) } \\
\text { Método de } \\
\text { estimación: } \\
\text { Componentes } \\
\text { principales con } \\
\text { rotación Varimax y } \\
\text { Oblimim }\end{array}$ & $\begin{array}{l}\text { Indicadores de } \\
\text { Ajuste del modelo: } \\
\text { chi-cuadrado, } \\
\text { RMSR y AGFI }\end{array}$ & $\begin{array}{l}\text { AFE: } 6 \text { factores } \\
\text { ( } 52 \% \text { de varianza } \\
\text { explicada) } \\
\text { AFC: Solución } \\
\text { de seis factores } \\
\text { presentó mejor } \\
\text { ajuste que } \\
\text { soluciones } \\
\text { alternativas }\end{array}$ \\
\hline México ${ }^{26}$ & $\begin{array}{l}\mathrm{n}=650 \\
\text { Muestra no } \\
\text { clínica. } \\
65 \% \text { mujeres } \\
15-53 \text { años } \\
\text { (M y DE no } \\
\text { reportadas) }\end{array}$ & $\begin{array}{l}\text { Versión adaptada } \\
\text { directamente } \\
\text { del original } \\
\text { revisado (edición } \\
\text { 1993) por los } \\
\text { investigadores. } \\
\text { Fue evaluada por } \\
10 \text { jueces expertos } \\
\text { y piloteada con } \\
\text { una muestra de } \\
\mathrm{n}=81 \\
21 \text { ítems }\end{array}$ & $\begin{array}{l}\text { n de factores: } \\
\text { No se reporta } \\
\text { Método de } \\
\text { estimación: No se } \\
\text { reporta, sólo se } \\
\text { informa rotación } \\
\text { Varimax }\end{array}$ & No se realizó & $\begin{array}{l}\text { AFE: Solución de } \\
\text { tres factores (no } \\
\text { se reporta \% de } \\
\text { varianza explicada } \\
\text { por el modelo) }\end{array}$ \\
\hline Argentina $^{7}$ & $\begin{array}{l}\mathrm{n}=550 \\
\text { Muestra no clínica } \\
68 \% \text { mujeres } \\
18-65 \text { años } \\
(\mathrm{M}=29,63 \\
\mathrm{DE}=0,32)\end{array}$ & $\begin{array}{l}\text { No explicitan } \\
\text { versión utilizada, } \\
\text { pero citan la } \\
\text { versión en inglés } \\
\text { revisada de Beck } \\
\text { et al. (1979) } \\
21 \text { ítems }\end{array}$ & $\begin{array}{l}\text { n de factores: } \\
\text { Criterio Kaiser } \\
\text { (eigenvalue } \geq 1 \text { ); } \\
\text { saturación } \geq 0,30 \\
\text { y scree test. No } \\
\text { especifican el } \\
\text { criterio decisivo. } \\
\text { Método de } \\
\text { estimación: } \\
\text { componentes } \\
\text { principales con } \\
\text { rotación promax }\end{array}$ & No se realizó & $\begin{array}{l}\text { AFE: Solución } \\
\text { de dos factores } \\
\text { relacionados, } \\
\text { ( } 27 \% \text { de varianza } \\
\text { explicada) } \\
\text { Solución final } \\
\text { elimina un ítem }\end{array}$ \\
\hline Puerto Rico ${ }^{28}$ & $\begin{array}{l}\mathrm{n}=351 \\
\text { Muestra no clínica } \\
69 \% \text { mujeres } \\
\text { Rango de edad } \\
\text { no reportado } \\
(\mathrm{M}=21,65 \\
\mathrm{DE}=4,03)\end{array}$ & $\begin{array}{l}\text { Versión adaptada } \\
\text { del original en } \\
\text { inglés } \\
22 \text { ítems }\end{array}$ & $\begin{array}{l}\text { n de factores: No } \\
\text { se reporta criterio. } \\
\text { Método de } \\
\text { estimación: } \\
\text { componentes } \\
\text { principales con } \\
\text { rotación Varimax }\end{array}$ & No se realizó & $\begin{array}{l}\text { AFE: Solución de } \\
\text { cuatro factores, } \\
\text { (49\% de varianza } \\
\text { explicada) }\end{array}$ \\
\hline
\end{tabular}


versión mexicana que presenta una solución de tres factores ${ }^{26}$, la versión argentina que obtuvo una solución bifactorial ${ }^{7}$, la versión puertorriqueña que obtuvo una solución de cuatro factore ${ }^{28}$ y la versión española que obtuvo una solución de seis factores ${ }^{27}$. Estas diferencias podrían entenderse por múltiples razones, tanto metodológicas como estadísticas.

En términos metodológicos, es importante considerar las discrepancias en los procedimientos de adaptación del instrumento, teniendo en cuenta que los inventarios se han elaborado adaptando diversas versiones del original en inglés, y que incluso presentan variaciones en el número de ítems (versiones de 19, 21 y 22 ítems). Otro aspecto metodológico que debe contemplarse es el tipo de muestra con que se han realizado los estudios. El presente estudio incluyó una muestra compuesta tanto por personas con diagnóstico clínico de depresión como por personas sin diagnóstico conocido de trastornos mentales, mientras que en las otras versiones aplicadas a población hispanoparlante solo fueron incluidas muestras no clínicas.

En relación a los procedimientos estadísticos, se observan amplias diferencias en los métodos utilizados para evaluar la validez del instrumento. En primer lugar, las versiones argentina, puertorriqueña y española del instrumento efectúan un análisis de componentes principales (ACP), es decir, se extraen componentes para reducir al máximo las dimensiones del instrumento, pero no se extraen factores en el estricto significado del término $^{9,29}$. Al realizar el AFE, la varianza de un ítem es particionada en una varianza compartida y en una varianza error, siendo esta varianza en común la que permite dar cuenta de la estructura factorial a la base del instrumento, en cambio el ACP no discrimina entre estas dos fuentes de varianza, pudiendo -bajo determinadas circunstanciasgenerar soluciones no estables y/o sobreestimar la varianza explicada por los factores ${ }^{29}$.

Así, si bien la estructura factorial del BDIIA puede variar en número y contenido de los factores, esta inestabilidad puede entenderse no solo por las diferencias en los análisis estadísticos realizados, sino también por la naturaleza de las muestras estudiadas. Ibáñez, Peñate y González (1997) sugieren que es probable que muestras de sujetos depresivos tiendan a proporcionar una estructura más homogénea, como la solución encontrada en este estudio, considerando que se trabajó tanto con personas con diagnóstico clínico de depresión y sin diagnóstico conocido de trastornos mentales ${ }^{27}$.

Por otro lado, a pesar de que la muestra utilizada en el presente estudio corresponde en mayor porcentaje a mujeres, la estructura del instrumento presentó estabilidad entre hombres y mujeres, por lo que la estructura unifactorial da cuenta en forma adecuada de la sintomatología depresiva en población de ambos sexos.

Además, se calculó el puntaje de corte óptimo para esta muestra, utilizando dos métodos. Ambos métodos entregaron puntajes diferentes entre ellos y distintos -además-al puntaje de corte propuesto en la solución original del instrumento ${ }^{10}$. Recomendamos utilizar el punto de corte 13/14 propuesto por el índice de Youden, considerando que este método maximiza tanto la sensibilidad como la especificidad de la clasificación ${ }^{20}$.

Para concluir, se puede señalar que a la luz de los resultados obtenidos, la versión chilena del BDI-IA presenta adecuadas propiedades psicométricas como instrumento de evaluación del síndrome depresivo, lo que permite recomendar su elección como instrumento de cribado para clasificar a los sujetos como deprimidos o no deprimidos, tanto en contextos clínicos como de investigación. Por otro lado, dado que este instrumento no requiere pago ni autorización por parte de sus autores para ser utilizado y que ha mostrado ser una herramienta efectiva de detección de depresión en atención primaria ${ }^{30}$, podrá ser de gran utilidad en contextos de salud pública, tanto para su uso en población general, como en pacientes ya diagnosticados.

\section{Referencias}

1. Izquierdo A. Validación de la versión española del BDIFS: un instrumento para la detección de la depresión en atención primaria [Tesis Doctoral]. Madrid: Facultad de Psicología, Universidad Complutense de Madrid; 2011 [Consultado el 19 de junio de 2015]. http://eprints.ucm. es/14539/1/T33361.pdf

2. Martínez V, Rojas G, Fritsch R. Programas de prevención de la depresión en adolescentes en el sistema escolar: Una revisión de la literatura. Rev GPU 2008; 4 (2): 206-13.

3. Ministerio de Salud, Chile. Encuesta Nacional de Salud ENS Chile 2009-2010; 2011 [Consultado el 19 de junio 
de 2015]. Disponible en http://web.minsal.cl/portal/url/ item/bcb03d7bc28b64dfe040010165012d23.pdf

4. Vicente B, Rioseco P, Saldivia S, Kohn R, Torres, S. Estudio chileno de prevalencia de patología psiquiátrica (DSM-III-R/CIDI) (ECPP). Rev Med Chile 2002; 130 (5): 527-36.

5. Subsecretaría de Salud Pública, Ministerio de Salud, Chile. Informe final estudio de carga de enfermedad y carga atribuible 2007; 2008. [Consultado el 19 de junio de 2015]. Recuperado de http://www.cienciasdelasalud-udla.cl/portales/tp76246caadc23/uploadImg/File/ Informe-final-carga-Enf-2007.pdf

6. Departamento de Estudios y Desarrollo, Superintendencia de Salud, Chile. Análisis de Licencias Médicas y Gasto por Subsidio por Incapacidad Laboral, Año 2010; 2012. [Consultado el 19 de junio de 2015]. Disponible en http://www.supersalud.gob.cl/documentacion/569/ articles-8039_recurso_1.pdf

7. Posada MC. Investigación psicométrica del Inventario de Depresión de Beck. Datos normativos en la ciudad de Mar del Plata. Perspect Psicol 2005; 2 (1): 58-63.

8. Beck AT, Steer RA, Carbin MG. Psychometric properties of the Beck Depression Inventory: Twenty-five years of evaluation. Clinical Psychology Rev 1988; 8 (1): 77-100.

9. Floyd FJ, Widaman KF. Factor analysis in the development and refinement of clinical assessment instruments. Psychol Assess 1995; 7 (3): 286-99.

10. Beck AT, Ward CH, Mendelson M, Mock J, Erbaugh JK. An inventory for measuring depression. Arch Gen Psychiatry 1961; 4 (6): 561-71.

11. Sanz J, Vázquez C. Fiabilidad, validez y datos normativos del Inventario para la Depresión de Beck. Psicothema 1998; 10 (2): 303-18.

12. Fabrigar LR, Wegener DT, MacCallum RC, Strahan EJ. Evaluating the use of exploratory factor analysis in psychological research. Psychol Methods 1999; 4 (3): 272-99.

13. O'Connor BP. SPSS and SAS programs for determining the number of components using parallel analysis and Velicer's MAP test. Behav Res Meth Instrum Comput 2000; 32 (3): 396-402.

14. Jackson DL, Gillaspy Jr JA, Purc-Stephenson R. Reporting practices in confirmatory factor analysis: an overview and some recommendations. Psychol Methods 2009; 14 (1): 6-23.

15. Batista-Foguet JM, Coenders G, Alonso J. Análisis factorial confirmatorio. Su utilidad en la validación de cuestionarios relacionados con la salud. Med Clin 2004; 122 (1): 21-7.

16. Browne MW, Cudeck R. Alternative ways of assessing model fit. En: Bollen KA, Long JS (Eds.) Testing structural equation models. Beverly Hills, CA, USA: Sage; 1993. p. 136-62.

17. Hu LT, Bentler PM. Cutoff criteria for fit indexes in covariance structure analysis: Conventional criteria versus new alternatives. Structural Equation Modeling: A Multidisciplinary Journal 1999; 6 (1): 1-55.

18. Fawcett T. An introduction to ROC analysis. Pattern Recognition Letters 2006; 27: 861-74.

19. Youden WJ. Index for rating diagnostic tests. Cancer 1950; 3: 32-5.

20. Perkins NJ, Schisterman EF. The inconsistency of "optimal" cutpoints obtained using two criteria based on the Receiver Operating Characteristic curve. Am J Epidemiol 2006; 163 (7): 670-5.

21. R Development Core Team. A language and environment for statistical computing. Vienna: R Foundation for Statistical Computing; 2014.

22. Robin X, Turck N, Hainard A, Tiberti N, Lisacek F, Sanchez JC, et al. pROC: An open-source package for $\mathrm{R}$ and $\mathrm{S}+$ to analyze and compare ROC curves. BMC Bioinformatics 2011; 12: 77-84.

23. Muthén LK, Muthén BO. Mplus User's Guide. $7^{\circ}$ ed. Los Angeles, CA: Muthén \& Muthén; 1998-2012.

24. Stevens JP. Applied multivariate statistics for the social sciences (4th Ed). Mahwah, New Jersey: Lawrence Erlbaum Associates; 2002.

25. Beck AT, Rush AJ, Shaw BF, Emery G. Cognitive Therapy of Depression. USA, New York: Guilford Press; 1979.

26. Jurado S, Villegas ME, Méndez L, Rodríguez F, Loperena V, Varela, R. La estandarización del Inventario de Depresión de Beck para los residentes de la Ciudad de México. Salud Mental 1998; 21(3): 26-31.

27. Ibáñez I, Peñate W, González M. La estructura factorial del Inventario de Depresión de Beck. Psicol Conduct 1997; 5 (1): 71-91.

28. Bonilla J, Bernal G, Santos A, Santos D. A revised Spanish version of the Beck Depression Inventory: Psychometric properties with a Puerto Rican sample of college students. J Clin Psychol 2004; 60 (1): 119-30.

29. Costello AB, Osborne JW. Best practices in exploratory factor analysis: Four recommendations for getting the most from your analysis. PARE 2005; 10 (7): 1-9. 